\title{
Improved nowcasting of precipitation based on convective analysis fields
}

\author{
M. Steinheimer ${ }^{1}$ and T. Haiden ${ }^{2}$ \\ ${ }^{1}$ Institute for Meteorology and Geophysics, University of Vienna, Austria \\ ${ }^{2}$ Central Institute for Meteorology and Geodynamics, Vienna, Austria
}

Received: 8 July 2006 - Revised: 18 January 2007 - Accepted: 30 January 2007 - Published: 26 April 2007

\begin{abstract}
The high-resolution analysis and nowcasting system INCA (Integrated Nowcasting through Comprehensive Analysis) developed at the Austrian national weather service provides three-dimensional fields of temperature, humidity, and wind on an hourly basis, and two-dimensional fields of precipitation rate in $15 \mathrm{~min}$ intervals. The system operates on a horizontal resolution of $1 \mathrm{~km}$ and a vertical resolution of 100-200 m. It combines surface station data, remote sensing data (radar, satellite), forecast fields of the numerical weather prediction model ALADIN, and high-resolution topographic data. An important application of the INCA system is nowcasting of convective precipitation. Based on fine-scale temperature, humidity, and wind analyses a number of convective analysis fields are routinely generated. These fields include convective boundary layer (CBL) flow convergence and specific humidity, lifted condensation level (LCL), convective available potential energy (CAPE), convective inhibition (CIN), and various convective stability indices. Based on the verification of areal precipitation nowcasts it is shown that the pure translational forecast of convective cells can be improved by using a decision algorithm which is based on a subset of the above fields, combined with satellite products.
\end{abstract}

\section{Introduction}

There is an increasing demand for automated high-quality very-short-range forecasts and nowcasts not just of precipitation but also temperature, wind, humidity, cloudiness, or global radiation. Real-time flood-warning systems based on detailed hydrological modeling require meteorological input on small scales and at high update frequencies. Highresolution road weather forecasts become increasingly important both for winter road management and for transporta-

Correspondence to: T. Haiden

(thomas.haiden@zamg.ac.at) tion planning. In response to these requirements the analysis and nowcasting system INCA (Integrated Nowcasting through Comprehensive Analysis) has been developed at ZAMG (Central Institute for Meteorology and Geodynamics, Vienna, Austria). Section 2 gives a short overview of its general characteristics, data sources, and the analysis and nowcasting methods used.

Most existing observation-based forecasting systems have been designed for the prediction of precipitation and convective phenomena (Browning and Collier, 1989; Li et al., 1995; Hand, 1996; Golding, 1998; Pierce et al., 2000). During the World Weather Research Program (WWRP) Forecast Demonstration Project (FDP) of the 2000 Sydney Olympics several systems were tested and compared (Pierce et al., 2004). A wind analysis and nowcasting system was also tested and evaluated during that project (Crook and Sun, 2004). The work, however, was not primarily focused on the wind field as such but on its use for the prediction of the initiation and development of deep convection (Wilson and Schreiber, 1986; Wilson et al., 2004). Similarly, analysis and nowcasting of near-surface temperature (Sun and Crook, 2001) has been of interest mainly in the prediction of convective developments rather than as a forecast field in itself.

These systems use different methods of determining precipitation movement such as area tracking, individual cell tracking, and numerical weather prediction (NWP) model winds. Two of the systems, namely GANDOLF (MetOffice, Exeter, UK) and ANC (NCAR, Boulder, CO, USA) also have convective evolution and initiation capability (Wilson et al., 2004). In the GANDOLF system, convective cells are classified into different stages of development. Based on a conceptual model of storm evolution, future states are predicted. New ("daughter") cells can be initiated close to existing cells if the boundary layer convergence predicted by the NWP model is sufficiently strong. The ANC predicts cell initiation and evolution based on the interaction of existing storms and cumulus clouds with boundary layer convergence

Published by Copernicus GmbH on behalf of the European Geosciences Union. 


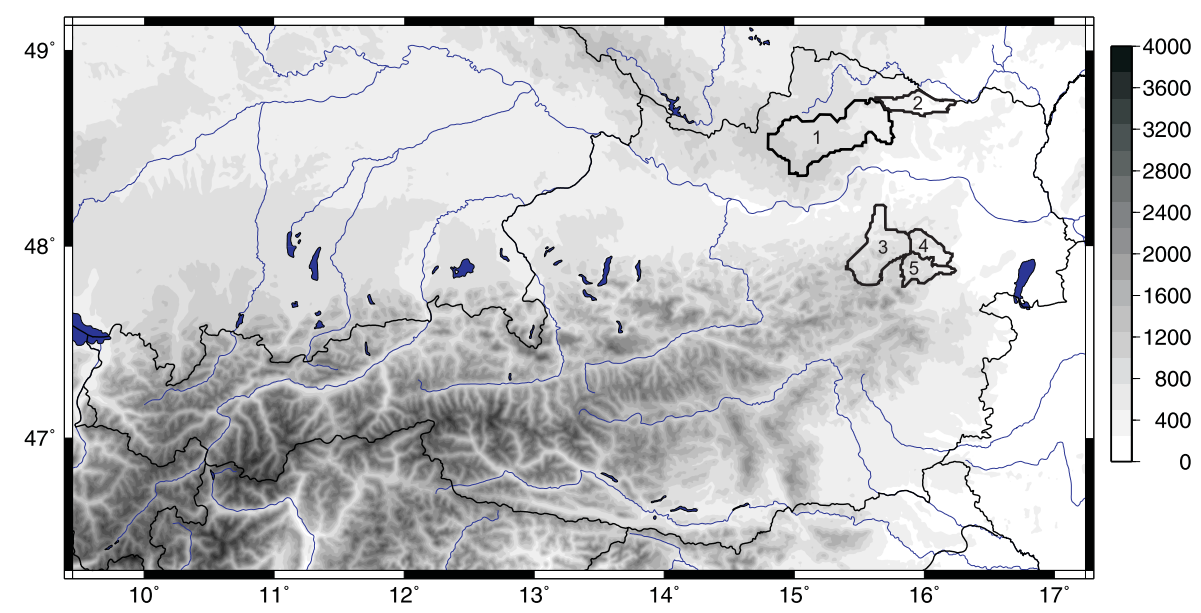

Fig. 1. INCA domain and topography (meter above mean sea level). Domain size is $600 \times 350 \mathrm{~km}$, horizontal resolution is $1 \mathrm{~km}$. River catchments referred to in the study are Kamp (1), Pulkau (2), Traisen (3), Triesting (4), and Piesting (5).

lines observed by radar and predicted by NWP wind field characteristics. The main findings with regard to convective cell prediction in the Sydney FDP can be summarized as follows (Wilson et al., 2004):

1. Predictive skill above pure translation occurs when boundary layer convergence lines can be identified and used to nowcast cell evolution.

2. For nowcasts beyond $60 \mathrm{~min}$, boundary characteristics are more important for storm initiation than early detection of cumulus clouds.

For the development of a cell initiation and evolution module in INCA, described in Sect. 3, these results served as a guideline. They also indicate the importance of a good boundary-layer wind field analysis. In Austria's alpine terrain, boundary layer convergence lines are to a large degree related to topography, which adds a deterministic component to cell initiation (Haiden, 2001). With the current version of the INCA wind field analysis the ability to correctly detect these convergence lines critically depends on the skill of the ALADIN $^{1}$ model in predicting them, and on the surface station network to represent them. Case studies are carried out to determine to what extent this is in fact the case. However, none of the systems used in the Sydney FDP used convective analysis fields such as CAPE or CIN, so it is interesting to investigate what additional benefit can be gained from those. Results of the case studies are discussed in Sect. 4.

\footnotetext{
${ }^{1}$ ALADIN is a limited area weather prediction model (LAM). In this study ALADIN-VIENNA is used, that runs operationally at ZAMG. (horizontal resolution: $9.6 \mathrm{~km}$ ).
}

\section{The INCA system}

The forecast of an NWP model is trilinearly interpolated to the high-resolution $(1 \mathrm{~km})$ INCA grid, serving as a first guess. Differences between surface observations and the first guess are determined and spatially interpolated using inverse distance weighting (IDW) both in geometric and in physical space. In the case of temperature, for example, geometrical distance weighting is used in the horizontal, while in the vertical the distance weighting is performed in potential temperature $(\theta)$ space. The three-dimensional "distance" between INCA gridpoint $(i, j, m)$ and the $k$-th surface station is given by

$$
\begin{aligned}
& r_{i j m k}= \\
& \sqrt{\left(x_{k}-x_{i}\right)^{2}+\left(y_{k}-y_{j}\right)^{2}+c^{2}\left(\theta_{k}^{\mathrm{NWP}}-\theta_{i j m}^{\mathrm{NWP}}\right)^{2}},
\end{aligned}
$$

where the parameter $c$ has the dimension of an inverse temperature gradient. The interpolated three-dimensional temperature difference field is obtained from

$\Delta \theta(i, j, m)=\frac{\sum_{k} \frac{\theta_{k}^{\mathrm{OBS}}-\theta_{k}^{\mathrm{NWP}}}{r_{i j m k}^{2}}}{\sum_{k} \frac{1}{r_{i j m k}^{2}}}$

and added to the NWP first guess, giving

$\theta^{\mathrm{INCA}}(i, j, m)=\theta^{\mathrm{NWP}}(i, j, m)+\Delta \theta(i, j, m)$.

In case of wind a relaxation algorithm is applied to the interpolated field in order to obtain mass-consistency. The current downscaling procedure in INCA is purely kinematic and cannot introduce dynamical flow effects such as mountain waves or foehn if these phenomena are not already present in the NWP field or the observations. The same applies to boundary layer convergence. Hence, the resulting convergence is sensitive to the quality of the first guess provided by 
the NWP. In areas with orographically induced convergence good results are achieved, as pointed out in Sect. 4.

INCA routinely analyzes three-dimensional fields with one hour update interval (temperature, humidity, wind), twodimensional fields with one hour update interval (global radiation, convective analysis fields) and two-dimensional fields with 15 minutes update interval (cloudiness, precipitation). Analyses and nowcasts of these fields have been operational since 2005.

The high resolution of $1 \mathrm{~km}$ is an essential characteristic of INCA. It enables the system to process locally influenced station observations, because at this resolution the actual elevation and exposition of most surface stations coincides reasonably well with the corresponding values on the numerical grid. In steep terrain the resolution is still not entirely adequate but it is an operationally feasible compromise for a domain size encompassing the entire area of Austria $(600 \times 350 \mathrm{~km}$, Fig. 1). Another reason for using a $1 \mathrm{~km}$ grid is that it corresponds to the resolution of the radar data used in INCA.

In the vertical, a $z$-system is used where $z$ is the height above the "valley-floor surface". In mountainous or hilly terrain, the valley-floors of adjacent valleys are generally found at comparable heights. Thus one may define a hypothetical surface that is smooth compared to the actual topography and connects major valley-floors (Haiden, 1998). This surface represents a useful local reference height for the $z$-System. The vertical resolution of INCA is currently equidistant at $\Delta z=200 \mathrm{~m}$. The system has 21 levels (surface included) parallel to the valley-floor surface, covering the lowest $4000 \mathrm{~m}$ above this surface. For the wind analysis a $z$-coordinate with horizontal levels and $\Delta z=125 \mathrm{~m}$ is used. The irregular shape and reduced volume of grid elements intersecting the terrain is taken into account using the shaved element approach (Steppeler et al., 2002).

ALADIN forecast fields are used as a first guess for the three-dimensional INCA analyses (temperature, humidity, wind). The NWP fields are 1-hourly, at a horizontal resolution of $9.6 \mathrm{~km}$, with 45 levels in the vertical. Two ALADIN forecast runs per day are performed (00:00 UTC, 12:00 UTC). Post-processed fields from these runs are available at about 05:00 UTC and 17:00 UTC. ALADIN forecast fields used in INCA are geopotential, temperature, relative humidity, $u-, v-, w$ - wind components (3-d fields), $2 \mathrm{~m}$ temperature and relative humidity, $u-, v-10 \mathrm{~m}$-wind components, precipitation, total cloudiness, and low cloudiness (2-d fields).

The most important observational data for the INCA system are surface stations. ZAMG operates a network of $\sim 140$ automated stations (TAWES) across the country. In the vertical, they span most of the topographic range in Austria, with highest stations Brunnenkogel $(3440 \mathrm{~m})$, and Sonnblick $(3105 \mathrm{~m})$. Although the distribution of stations is biased towards valley locations, there is a sufficient number of mountain stations to construct three-dimensional correction fields
Table 1. Convective nowcast algorithm decision criteria and threshold values for cell initiation, intensification, and weakening.

\begin{tabular}{|c|c|c|c|c|}
\hline \multicolumn{5}{|c|}{ Cell initiation } \\
\hline CAPE & $>$ & $\mathrm{CAPE}_{\mathrm{ini}}$ & $=$ & $100 \mathrm{~J} \mathrm{~kg}^{-1}$ \\
\hline MOCON & $>$ & $\mathrm{MOCON}_{\text {ini }}$ & $=$ & $2 \times 10^{-6} s^{-1}$ \\
\hline VIS & $>$ & $\mathrm{VIS}_{\text {ini }}$ & $=$ & 0.5 \\
\hline $\mathrm{CT}$ & $\neq$ & $\mathrm{CT}_{\text {excl }}$ & $=$ & {$[3,4,1516]$} \\
\hline CIN & $<$ & $\mathrm{CIN}_{\mathrm{ini}}$ & $=$ & $200 \mathrm{~J} \mathrm{~kg}^{-1}$ \\
\hline DTTRIG & $>$ & DTTRIG $_{\text {ini }}$ & $=$ & $-2 \mathrm{~K}$ \\
\hline \multicolumn{5}{|c|}{ Cell intensification } \\
\hline CAPE & $>$ & CAPE $_{\text {int }}$ & $=$ & $50 \mathrm{~J} \mathrm{~kg}^{-1}$ \\
\hline MOCON & $>$ & $\mathrm{MOCON}_{\text {int }}$ & $=$ & $2 \times 10^{-6} s^{-1}$ \\
\hline $\mathrm{CT}$ & $\neq$ & $\mathrm{CT}_{\mathrm{excl}}$ & $=$ & {$[3,4,1516]$} \\
\hline $\mathrm{CIN}$ & $<$ & $\mathrm{CIN}_{\text {int }}$ & $=$ & $200 \mathrm{~J} \mathrm{~kg}^{-1}$ \\
\hline DTTRIG & $>$ & DTTRIG $_{\text {int }}$ & $=$ & $-2 \mathrm{~K}$ \\
\hline $\mathrm{RR}$ & $>$ & $\mathrm{RR}_{\text {int }}$ & $=$ & $0.2 \mathrm{~mm} \mathrm{~h}^{-1}$ \\
\hline \multicolumn{5}{|c|}{ Cell weakening } \\
\hline CAPE & $<$ & $\mathrm{CAPE}_{\text {weak }}$ & $=\min$ & $\left(\begin{array}{c}\overline{\mathrm{CAPE}}^{>0} \\
100 \mathrm{~J} \mathrm{~kg}^{-1}\end{array}\right.$ \\
\hline MOCON & $<$ & MOCON $_{\text {weak }}$ & $=$ & $0.0 \mathrm{~s}^{-1}$ \\
\hline RR & $>$ & $\mathrm{RR}_{\text {weak }}$ & $=$ & $0.0 \mathrm{~mm} \mathrm{~h}^{-1}$ \\
\hline
\end{tabular}

to the NWP model output on the basis of these observations. Radar data used in INCA is a 2-d composite of four radar stations with ground clutter already removed. The data has 14 intensity categories and a time resolution of $5 \mathrm{~min}$. Due to the mountainous character of the country, radar data is of limited use in many areas in western Austria, especially during wintertime when precipitation can originate from shallow cloud systems. The Meteosat 2nd Generation (MSG) satellite provides a number of new fields as well as a higher resolution than previous Meteosat imagery. The satellite products used in INCA are visible brightness and "Cloud Type" which differentiates between different cloud levels (low, medium, high) and different degrees of opaqueness.

\section{Convective nowcast algorithm}

The INCA translational precipitation nowcast is an observation-based extrapolation that uses motion vectors determined from consecutive analyses with a correlation technique. The size of the correlation quadrangle is $100 \mathrm{~km}$. Spurious correlations implying unrealistically large translation speeds are meteorologically filtered by comparison with ALADIN wind fields at 500 und $700 \mathrm{hPa}$. The filtering is done using the condition

$$
\left|\boldsymbol{V}_{\mathrm{KORR}}\right|+\left|\boldsymbol{V}_{\mathrm{KORR}}-\boldsymbol{V}_{\mathrm{ALA}}\right| \leq\left|\boldsymbol{V}_{\mathrm{ALA}}\right|+2 \Delta,
$$


where $\boldsymbol{V}_{\mathrm{KORR}}$ is the motion vector derived by correlation analysis, $\boldsymbol{V}_{\mathrm{ALA}}$ is the ALADIN 500 or $700 \mathrm{hPa}$ wind (whichever is closer to $\boldsymbol{V}_{\mathrm{KORR}}$ ), and $\Delta$ is a prescribed wind speed scale which determines the amount of deviation permitted between $\boldsymbol{V}_{\mathrm{KORR}}$ and $\boldsymbol{V}_{\mathrm{ALA}}$. Operationally, the value $\Delta=5 \mathrm{~ms}^{-1}$ is used. Equation (4) defines an elliptic area aligned with the vector $\boldsymbol{V}_{\text {ALA }}$. Verification of both point and areal precipitation forecasts shows that the INCA translational nowcast is superior to NWP forecasts over the first $2-4 \mathrm{~h}$.

In order to improve the convective precipitation forecast over that of a simple translation, the evolution (initiation, intensification, weakening) of convective cells must be assessed. Once every hour INCA routinely generates the convective analysis fields of lifted condensation level (LCL), level of free convection (LFC), convective available potential energy (CAPE), convective inhibition (CIN), Showalter index (SWI), lifted index (LI), trigger temperature deficit (DTTRIG), equivalent potential temperature $\left(\theta_{e}\right)$, boundarylayer convergence $(\mathrm{CON})$ and boundary-layer humidity convergence (MOCON). During the period of algorithm development several different combinations of these fields were tested. The ones that proved most useful and were ultimately used are indicated by italics.

The basic concept of the INCA convective nowcast algorithm is to classify each gridpoint as either "convective" (using the condition that CAPE $>50 \mathrm{~J} \mathrm{~kg}^{-1}$ within a certain distance from the gridpoint) or "non-convective". For each convective gridpoint it is tested whether the conditions for cell initiation, cell intensification, or cell weakening are fulfilled (Table 1). In addition to the above fields, Table 1 contains criteria based on MSG satellite data, namely visible brightness and cloud type information:

- Visible brightness (VIS) is used to identify areas where non-precipitating cumulus convection is already present. Only in these areas cell initiation is allowed. VIS is scaled to its domain maximum value at a given time to allow the use of a constant threshold value (Table 1) throughout the daytime diurnal cycle. The current algorithm is unable to predict initiation after sunset.

- Cloud Type (CT) is used to exclude those areas from initiation and intensification where obviously no convective cloudiness is present. This applies to following types: cloud free land snow $(\mathrm{CT}=3)$, cloud free sea snow/ice $(\mathrm{CT}=4)$, high semi-transparent thin clouds $(\mathrm{CT}=15)$, high semi-transparent thick clouds $(\mathrm{CT}=16)$.

An additional condition, that the precipitation rate (RR) must be greater than a certain threshold value, had to be applied in order to avoid intensification of very small precipitation rates that are sometimes generated in the analysis as a result of station interpolation.
The convective nowcast is created as follows:

- The analyzed precipitation field is moved according to the motion vectors for the length of one time step $\Delta t$.

- The new intensity is calculated at gridpoints where the criteria of Table 1 are fulfilled using Eq. (6); otherwise the intensity is left unchanged.

- The modified field is moved according to the motion vectors for the length of another time step and again the intensity is updated at the appropriate gridpoints.

- This procedure is repeated till the end of the nowcast period.

All three types of intensity changes (initiation, intensification, and weakening) are modeled as a Gaussian variation in time

$I(t)=I_{\mathrm{MAX}} \exp \left\{-[\underbrace{\left(t-t_{\mathrm{MAX}}\right)}_{t_{\mathrm{REL}}} / \tau]^{2}\right\}$,

where the three parameters $I_{\mathrm{MAX}}$ (maximum rainfall rate), $t_{\text {MAX }}$ (time of maximum rainfall rate) or rather $t_{\text {REL }}$ (time of maximum rainfall rate relative to time $t$ ), and $\tau$ (cell evolution time-scale) must be determined.

The resulting precipitation intensity at time $t+\Delta t$ is then given by

$I(t+\Delta t)=I_{\mathrm{MAX}} \exp \left\{-\left[\left(\Delta t+t_{\mathrm{REL}}\right) / \tau\right]^{2}\right\}$.

Maximum cell intensity is parameterized as a function of specific humidity $q$ (at valley-floor level) and CAPE in the form

$I_{\mathrm{MAX}}=c_{1} \rho q \sqrt{\mathrm{CAPE}}$,

where $\rho=1 \mathrm{~kg} \mathrm{~m}^{-3}$, and the nondimensional coefficient $c_{1}=1 / 90$.

In the case of initiation and intensification $\tau$ is set to the constant value $\tau_{G}=1800$ seconds.

The time of maximum intensity of a newly initiated cell relative to time $t$ is set to $t_{\mathrm{REL}}=-2 \tau_{G}$. For cell intensification $t_{\mathrm{REL}}$ is derived from the ratio of maximum intensity to intensity at time $t$ by inverting Eq. (6) and setting $\Delta t=0$, which gives

$t_{\mathrm{REL}}=-\tau_{G} \sqrt{\ln \left[I_{\mathrm{MAX}} / I(t)\right]}$,

where $I_{\mathrm{MAX}}$ is parameterized using Eq. (7).

In the case of cell weakening it is assumed that $t_{\mathrm{REL}}=0$, $I_{\mathrm{MAX}}=I(t)$. The timescale of weakening (in units of seconds) is parameterized based on moisture convergence

$\tau_{D}=-\frac{c_{2}}{\mathrm{MOCON}}$,

where $c_{2}=9 \times 10^{-4}$. In addition, $\tau_{D}$ is limited by the constraint $1800 \leq \tau_{D} \leq 3600$ seconds. 

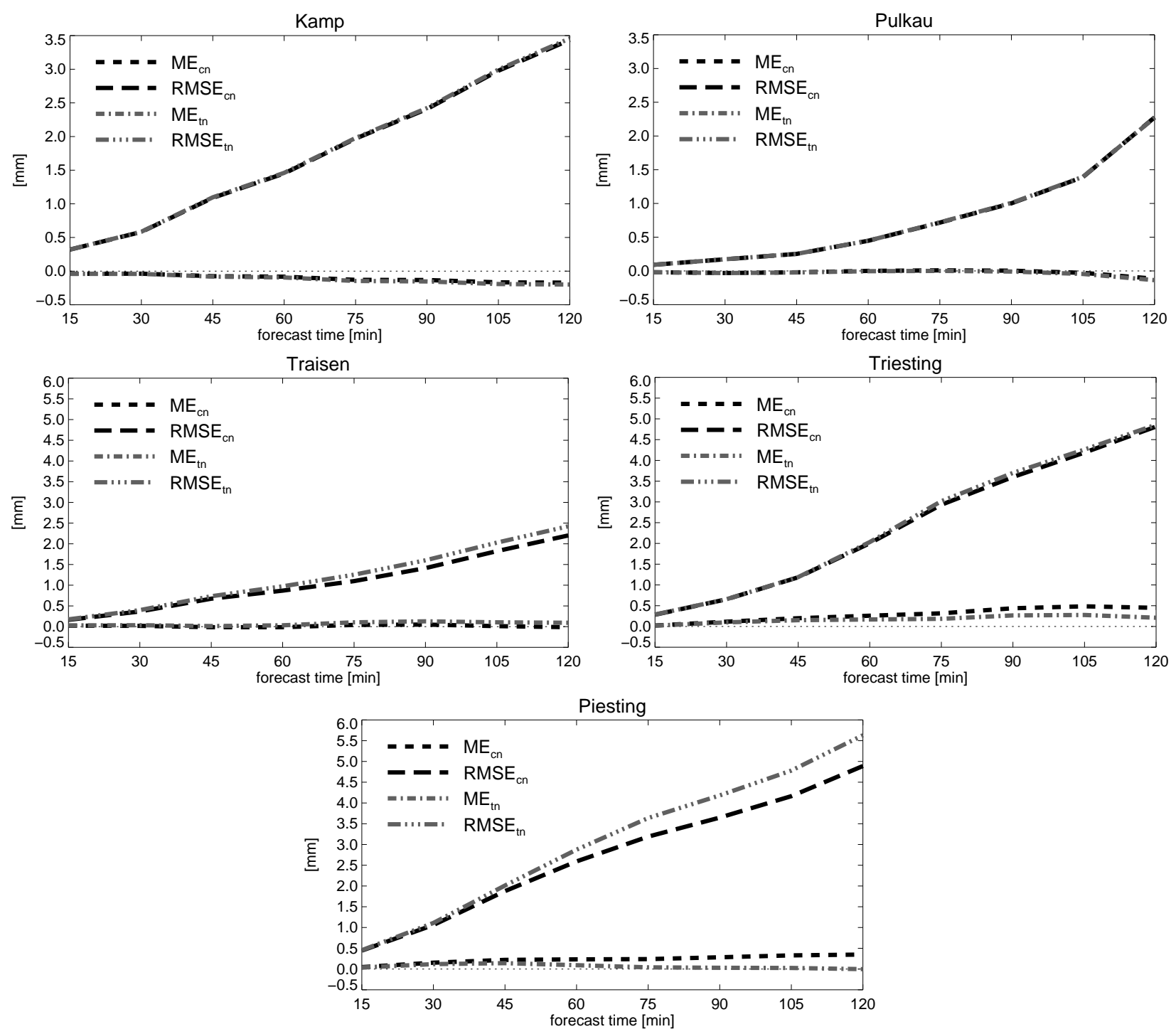

Fig. 2. Verification results for the non-alpine catchments Kamp and Pulkau, and the alpine catchments Traisen, Triesting, Piesting. Shown are the root-mean-square error (RMSE) and bias (ME) of areal precipitation nowcasts as a function of forecast time for the convective nowcast "cn" and the pure translational nowcast "tn".

\section{Results and discussion}

Verification of the convective nowcast algorithm is performed for five small river catchments (Fig. 1) in different parts of the province of Lower Austria with terrain ranging from hilly (Kamp, Pulkau) to moderately alpine (Traisen, Triesting, Piesting). Catchment sizes range from $250 \mathrm{~km}^{2}$ (Piesting) to $1500 \mathrm{~km}^{2}$ (Kamp). It was decided to verify areal rather than point forecasts because of their greater relevance to hydrological applications and because we did not want to penalize small location errors of the order of a few $\mathrm{km}$. The forecast range considered is +15 to $+120 \mathrm{~min}$, verification measures are root-mean-square error (RMSE) and bias (or mean error, ME) of accumulated precipitation, computed against INCA analyses. Results of the convective nowcast algorithm are compared to those obtained by pure translation. The verification period consists of all forecasts made at full hours between 11:00 and 18:00 UTC for those 10 days of the year 2005 which had the highest convective rainfall amounts in Lower Austria. All days fell within the period May-July.

When interpreting the verification results of the convective nowcast it must be kept in mind that a systematic improvement above pure translation is generally hard to achieve. By predicting intensity changes, in particular in the case of initiation and intensification, the convective nowcast takes a greater "risk" than the translation. Our attempt was to design 
an algorithm that gives significant improvements in individual cases without worsening the overall skill. On the other hand, the verification periods considered here cannot be considered entirely independent since they contain cases that have been used in the design of the algorithm. Thus we expected to see improvements at least in some of the areas.

Figure 2 shows the RMSE and ME for the translational and the convective nowcast. In the non-alpine areas the results are essentially neutral. Analysis of individual cases in these areas shows a number of days where there is in fact an improvement due to the convective nowcast but this is compensated by worsening on other days. Results for the alpine areas show a significant improvement in terms of RMSE for Piesting and Traisen, and rather neutral behaviour for Triesting. In the former two areas there are also individual cases where the convective nowcast algorithm has a larger error than the translational algorithm, but overall the improvements dominate. One reason why the improvement is more pronounced in the alpine areas may be that, due to orographic effects, the moisture convergence is more predictable and thus better represented in the ALADIN wind field that is used as a first guess in the INCA wind analysis. This assumption is based on the observation of strong moisture convergence in the region of our alpine verification areas that are well-known climatological "hot spots" for orographically triggered convective developments. The ME tends to increase slightly in areas where the RMSE is reduced. However, this is not always the case, as shown by the results for the Traisen catchment. With regard to differences in error magnitude between catchments it is important to note that they do not indicate a difference in forecast skill but primarily reflect different precipitation amounts.

In order to confirm whether the verification results from 2005 can be generalized, an additional continuous verification was performed which covers all nowcasts made between 4 April 2006 and 16 May 2006 during a pre-operational test run of the system. In this case no individual days were selected, thus the sample contains a mixture of non-convective and convective cases. Again, no significant change in RMSE was found for the non-alpine areas, whereas improvements similar in extent to the 2005 verification were found for two alpine areas (Triesting, Piesting) with the third (Traisen) giving neutral results.

Based on a number of individual cases, Wilson et al. (2004) provide a qualitative verification of the convective nowcast algorithm in the ANC system for up to $+60 \mathrm{~min}$. They demonstrate that it outperforms the purely translational forecast in most cases but also point out that further algorithm development is needed. In contrast to INCA, the ANC obtains wind fields derived from Doppler radar as input. It is likely that such input would increase the skill of the INCA convective nowcast especially in lowland areas, where the current wind analysis based on NWP results and station data appears to be unable to resolve the convergence characteristics relevant to convective intensity changes. Pierce et al.
(2000) made a quantitative evaluation, and comparison with pure translation, for the GANDOLF system. In contrast to INCA, GANDOLF uses an object-oriented algorithm to extrapolate cell movement and intensity. For all non-frontal convective events of 1995-1996, results similar to the ones presented here were obtained in the sense that both improvements and worsening compared to pure translation was found for different catchments.

\section{Conclusions}

An approach to convective nowcasting is presented which is based on high-resolution analyses of some key convective diagnostics. It is found that the convective analysis fields most important for the nowcast are CAPE, CIN, moisture convergence, and trigger temperature deficit. The current version of the algorithm also requires visible satellite imagery in order to identify areas of incipient cell intensification and to avoid over-prediction of cell initiation. Verification of areal precipitation nowcasts for small catchments shows that on average the convective nowcast performs slightly better than the purely translational nowcast. The improvement is more pronounced in the alpine catchments studied, whereas it is marginal in the non-alpine areas. This difference in behaviour appears to be due to the more predictable character of the orographically induced moisture convergence field along the main alpine rim as compared to that over the lowlands.

There are many possible ways in which the current version of the algorithm could be improved. For example, it could be argued that the thresholds set for input fields like CAPE, CIN, DTTRIG and MOCON should not be constants but rather depend on the values of the other fields at the given instant and location. Also, it would be more physical in terms of an ingredient-based approach to use mass convergence instead of moisture convergence. For the mesoscale structures studied here, however, the convergence term dominates MOCON, making mass and moisture convergence fields look rather similar (Banacos and Schultz, 2005).

Probably the largest improvement could be gained by improved analyses of the primary fields from which the convective diagnostics are derived. The typical length scale of CAPE, CIN, and MOCON variations in the analysis is significantly larger than the scale of individual convective cells. The DTTRIG parameter, which is smaller in scale, is not yet sufficiently reliable. To deal with this drawback the convective nowcast algorithm currently uses visible satellite imagery to decide where new precipitation cells are initiated. That means that the current nowcast algorithm is unable to predict cell initiation in clear air. Further work on INCA will address this problem through improved spatial interpolation techniques for temperature, humidity and wind. 
Acknowledgements. The provincial government of Lower Austria supported the work done within this research.

Edited by: S. C. Michaelides and E. Amitai

Reviewed by: anonymous referees

\section{References}

Banacos, P. C. and Schultz, D. M.: The use of moisture flux convergence in forecasting convective initiation: historical and opeational perspectives, Wea. Forecast., 20, 351-366, 2005.

Browning, K. A. and Collier, C. G.: Nowcasting of precipitation systems, Rev. Geophys., 27, 345-370, 1989.

Crook, N. A. and Sun, J.: Analysis and forecasting of the low-level wind during the Sydney 2000 Forecast Demonstration Project, Wea. Forecast., 19, 151-167, 2004.

Golding, B. W.: Nimrod: A system for generating automated very short range forecasts, Meteorol. Appl., 5, 1-16, 1998.

Haiden, T.: Analytical aspects of mixed-layer growth in complex terrain. Preprints, Eighth Conference on Mountain Meteorology, Amer. Meteorol. Soc., Flagstaff, Arizona, 368-372, 1998.

Haiden, T.: High-resolution forecasts of mountain cumulus, Proceedings, 22nd EWGLAM/7th SRNWP Meeting, Toulouse, France, 134-138, 2001
Hand, W. H.: An object-oriented technique for nowcasting heavy showers and thunderstorms, Meteorol. Appl., 3, 31-41, 1996.

Li, L., Schmid, W., and Joss, J.: Nowcasting of motion and growth of precipitation with radar over a complex topography, J. Appl. Meteorol., 34, 1286-1300, 1995.

Pierce, C. E., Hardaker, P. J., Collier, C. G., and Haggett, C. M.: GANDOLF: a system for generating automated nowcasts of convective precipitation, Meteorol. Appl., 7, 341-360, 2000.

Pierce, C. E., Ebert, E., Seed, A. W., Sleigh, M., Collier, C. G., Fox, N. I., Donaldson, N., Wilson, J. W., Roberts, R., and Mueller, C. K.: The nowcasting of precipitation during Sydney 2000: An appraisal of the QPF algorithms, Wea. Forecast., 19, 7-21, 2004.

Steppeler, J., Bitzer, H.-W., Minotte, M., and Bonaventura, L.: Nonhydrostatic atmospheric modeling using a z-coordinate representation, Mon. Wea. Rev., 130, 2143-2149, 2002.

Sun, J. and Crook, N. A.: Real-time low-level wind and temperature analysis using WSR-88D data, Wea. Forecast., 16, 117-132, 2001.

Wilson, J. W. and Schreiber, W. E.: Initiation of convective storms by radar-observed boundary layer convergence lines, Mon. Wea. Rev., 114, 2516-2536, 1986.

Wilson, J. W., Ebert, E. E., Saxen, T. R., Roberts, R. D., Mueller, C. K., Sleigh, M., Pierce, C. E., and Seed, A.: Sydney 2000 Forecast Demonstration Project: Convective storm nowcasting, Wea. Forecast., 19, 131-150, 2004. 